\title{
Síndrome de Bouveret. Resolución endóscopica y quirúrgica de cuatro casos clínicos
}

\author{
Armando Iñíguez, Jean Michel Butte, José Miguel Zúñiga, \\ Fernando Crovari, 0 svaldo Llanos. \\ Bouveret syndrome. \\ Report of four cases
}

Background: Bouveret syndrome is a duodenal obstruction caused by a biliary stone. Aim: To report patients with Bouveret syndrome. Material and Methods: Retrospective review of medical records of patients with Bouveret syndrome treated between 1976 and 2006. Results: We report three women and one man with a mean age of 62.5 years. None had a previous diagnosis of cholelithiasis. All presented with colicky pain in the right upper quadrant and vomiting, suggesting gastric retention. The diagnosis was suspected after a barium meal in two patients and with a CT scan on the other two. The endoscopical extraction or fragmentation of stones was attempted in three patients but was successful only in one. Three patients were operated and a stone impacted in the first portion of the duodenum was identified, along with a cholecystoduodenal fistula. A duodenostomy and stone extraction was performed. One patient was subjected to a cholecystectomy, fistula repair and gastrojejunoanastomosis. No patient died and all were discharged within 8 to 12 days after surgery. Conclusions: Bouveret syndrome is an uncommon complication of cholelithiasis. Endoscopy can be diagnostic and therapeutic. Surgery is the other therapeutic option (Rev Méd Chile 2008; 136: 163-8).

(Key w ords: Duodenostomy; Duodenal obstruction; Gallstones)

Recibido el 6 de diciembre, 2006. Aceptado el 14 de agosto, 2007.

Departamento de Cirugía Digestiva, División de Cirugía, Facultad de Medicina, Pontificia Universidad Católica de Chile. Santiago de Chile.

$\mathrm{E}^{1}$ síndrome de Bouveret es una obstrucción duodenal producida por la migración de un cálculo vesicular a través de una fístula colecistoduodenal. Es un tipo de íleo biliar en el que la

Correspondencia a: Dr. Armando Iñíguez Cuadra. Departamento de Cirugía Digestiva, División de Cirugía, Facultad de Medicina, Pontificia Universidad Católica de Chile. Marcoleta 367, Santiago, Chile. Teléfonos: 56-2-3543462, 56-2-3543870. Fax: 56-2-6329620. E mail:ainiguez@uc.cl obstrucción se produce a nivel del bulbo duodenal. Fue descrito por primera vez en 1770 por Beaussier ${ }^{1}$. Más tarde, León Bouveret ${ }^{2}$, comunicó dos casos de retención gástrica secundaria a un cálculo impactado en el bulbo duodenal. Habitualmente se presenta en pacientes mayores de 60 años con síntomas iniciales inespecíficos que, en general, no reflejan la magnitud del proceso inflamatorio y de las alteraciones anatómicas subyacentes. Posteriormente se instala el cuadro clásico de dolor abdominal por distensión gástrica 
y vómitos de retención a repetición, producto de la impactación del cálculo en el duodeno ${ }^{3,4}$.

Clásicamente el diagnóstico se ha apoyado en el estudio de imágenes, como la radiografía de abdomen simple, que en un tercio de los enfermos presentan la triada de Rigler, sugerente de íleo biliar (signos de obstrucción intestinal, neumobilia y cálculo en posición inhabitual), y otros métodos como la ecografía, la tomografía computada de abdomen y la endoscopia digestiva alta. Esta última además se ha utilizado como método de tratamiento, con el objeto de disminuir los riesgos de la cirugía clásica ${ }^{1,4}$.

El objetivo de este trabajo es describir las características clínicas, diagnósticas y terapéuticas de un grupo de pacientes con síndrome de Bouveret.

\section{MATERIAL Y MÉTODO}

Se revisó en forma retrospectiva los registros clínicos de los pacientes tratados por un síndrome de Bouveret entre 1976 y 2006 en el Hospital Clínico de la Pontificia Universidad Católica de Chile.

En cada enfermo se registraron las características clínicas, el estudio diagnóstico, el tratamiento practicado, la evolución inmediata y tardía.

\section{RESULTADOS}

Caso 1. Paciente de 36 años de edad de sexo femenino, con antecedente de úlcera gástrica tratada médicamente. Consultó por cuadro de 15 días de evolución, de dolor abdominal tipo cólico, asociado a vómitos post prandiales a repetición y coluria fugaz. En forma retrospectiva, la paciente relataba dolor en hipocondrio derecho tipo cólico biliar, intermitente los últimos 6 meses. Al examen físico se constató una paciente hemodinámicamente estable, sin signos de deshidratación, ni sepsis y un abdomen sensible en hipocondrio derecho asociado a una masa palpable de aproximadamente $8 \mathrm{~cm}$. En la evaluación inicial con exámenes de laboratorio no se encontró elevación de los leucocitos, ni alteración de la función renal o ácido base. A través de una gastroduodenografía con contraste oral, se observó una obstrucción duodenal de origen no precisado, más una probable fístula colecistoduodenal. Se instaló una sonda nasogástrica que dio 1.600 cc en las primeras $24 \mathrm{~h}$. Sin un diagnóstico claro, se decidió realizar una laparotomía exploradora, la cual reveló un proceso inflamatorio en hipocondrio derecho, una fístula colecistoduodenal de $3 \mathrm{~cm}$ de diámetro y un cálculo de $5 \mathrm{~cm}$ enclavado en duodeno, el que fue extraído a través del duodeno, se efectuó una colecistectomía y reparación del defecto duodenal. La paciente resolvió su íleo al $2^{\circ}$ día postoperatorio, siendo realimentada al $3^{\text {er }}$ día postoperatorio. Fue dada de alta al día 9 postoperatorio.

Caso 2. Paciente de sexo masculino, 55 años. Sin antecedentes mórbidos, consultó por inicio de síntomas 12 días antes, consistentes en dolor tipo cólico abdominal en epigastrio e hipocondrio derecho, vómitos a repetición, ictericia y coluria. A su ingreso en urgencias se constató un paciente deshidratado, taquicárdico (102 x min), sin alteración de la presión arterial. Al examen físico abdominal, se palpaba el hipocondrio derecho sensible, sin masa. En el análisis de laboratorio se encontró un recuento de leucocitos normal, con creatinina plasmática de $1,4 \mathrm{mg} / \mathrm{dl}$, sin elevación del nitrógeno ureico. Se realizó una radiografía abdominal que resultó sin alteraciones, se realizó además una gastroduodenografía con contraste oral, que reveló una imagen sugerente de cálculo impactado en la primera porción del duodeno, sin visualizar un trayecto fistuloso, encontrando sí, medio de contraste en la vesícula biliar. Se efectuó una ecotomografía abdominal que visualizó un cálculo de localización adyacente a la vesícula biliar-duodeno o estómago. Se realizó una endoscopia, en la cual se observó un gran cálculo en la primera porción del duodeno, el cual no pudo ser extraído con canastillo de Dormia en un primer intento, ni en un segundo intento realizado a las 72 h. Se decidió una resolución por vía quirúrgica. En el intraoperatorio se encontró una fístula colecistoduodenal, se realizó extracción del cálculo $(8 \mathrm{x}$ $5 \times 4 \mathrm{~cm})$ a través del duodeno, procediendo al cierre duodenal, sin realizar colecistectomía ni reparación de la fístula. Posteriormente se efectuó una yeyunostomía de alimentación. El paciente recibió alimentación por la yeyunostomía, iniciando alimentación por vía oral a los 15 días, presentó infección de la herida operatoria que se 
manejó con antibióticos y curaciones, retirando la sonda de yeyunostomía a las 4 semanas postoperatorias.

Caso 3. Paciente de 83 años, sexo femenino, con antecedente de diabetes mellitus y limitación crónica del flujo aéreo (LCFA). Consultó por cuadro de dolor abdominal cólico en hipocondrio derecho de 2 días de evolución, vómitos, sin fiebre y sin ictericia. Ingresó estable, destacando al examen físico dolor en hipocondrio derecho, asociado a una masa palpable. No presentaba leucocitosis, pero sí una elevación de la proteína $C$ reactiva de $2,2 \mathrm{mg} / \mathrm{dl}$. Se efectuó una tomografía axial computarizada (TAC) de abdomen y pelvis que mostró imágenes compatibles con una fístula colecistoduodenal, una vesícula biliar de paredes engrosadas, con un lúmen ocupado por un gran cálculo. Por endoscopia, se observó un cálculo post pilórico impactado, inmóvil, que no pudo ser extraído con canastillo de Dormia después de dos intentos realizados con $72 \mathrm{~h}$ de diferencia entre sí. Se decidió, en consecuencia, efectuar una resolución quirúrgica, encontrándose en la laparotomía un gran proceso inflamatorio en hipocondrio derecho, procediéndose a realizar una duodenotomía y extrayendo un cálculo de $8 \times 4 \mathrm{~cm}$ de diámetro (Figura 1), evidenciándose una fístula colecistoduodenal de aproximadamente $6 \mathrm{~cm}$. Se efectuó posteriormente cierre duodenal, sin colecistectomía, ni reparación de la fístula. La paciente fue realimentada al $6^{\circ}$ día postoperatorio y fue dada de alta al día 16 postoperatorio, con una infección de herida operatoria que se resolvió con curaciones y antibióticos ajustados al antibiograma.

Caso 4. Paciente de sexo femenino, 76 años. Portadora de diabetes mellitus 2 (DM-2), dislipidemia e hipertensión arterial. Presentaba desde algunos meses dolor en hipocondrio derecho tipo cólico que cedía espontáneamente. Fue hospitalizada por un cuadro de 4 días de duración, de dolor abdominal difuso, mayor en hemiabdomen superior, de tipo cólico y vómitos frecuentes, afebril y sin ictericia. Se constató una paciente deshidratada, sin alteraciones hemodinámicas. Al examen físico se encontró una masa palpable en hipocondrio derecho, sensible. Entre los exámenes destacaba una leucocitosis de $12.700 \mathrm{x}$ $\mathrm{mm}^{3}$ y una proteína C reactiva (PCR) de $5,5 \mathrm{mg} / \mathrm{dl}$, una creatinina plasmática elevada de 1,52 $\mathrm{mg} / \mathrm{dl}$, con un nitrógeno ureico (BUN) de $57 \mathrm{mg} / \mathrm{dl}$ y una alcalosis metabólica hipoclorémica. Estudio de imágenes: ecotomografía abdominal, colelitiasis y neumobilia. TAC: cálculo impactado en la primera porción duodenal, con signos de retención gástrica y una probable fístula colecistoduodenal. Se realizó una endoscopia digestiva alta que mostró un cálculo biliar post pilónico impactado (Figura 2), móvil, de $3 \mathrm{~cm}$ de diámetro aproximadamente, el cual fue fragmentado y extraído con canastillo de Dormia. Se realimentó al día 4 post endoscopia y fue dada de alta al día 7 post procedimiento.

No se observó mortalidad ni complicaciones mayores, precoces ni tardías a 4 meses de seguimiento promedio (1-12 meses), tanto en los casos

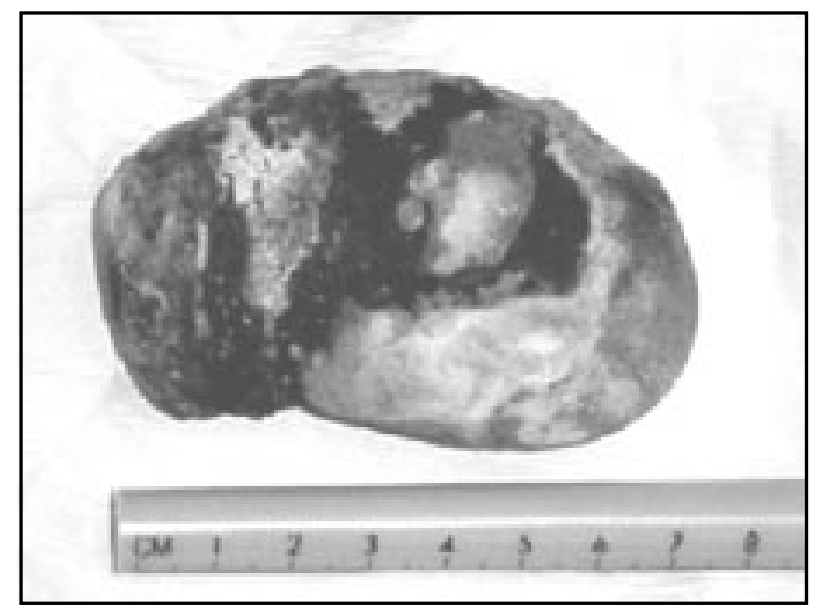

Figura 1. Cálculo extraído por vía quirúrgica. 
Figura 2. Endoscopia digestiva alta: se observa un cálculo biliar en la primera porción del duodeno, durante una maniobra de extracción con un canastillo de Dormia (AP =antro pilórico, P =píloro, CB =cálculo biliar).

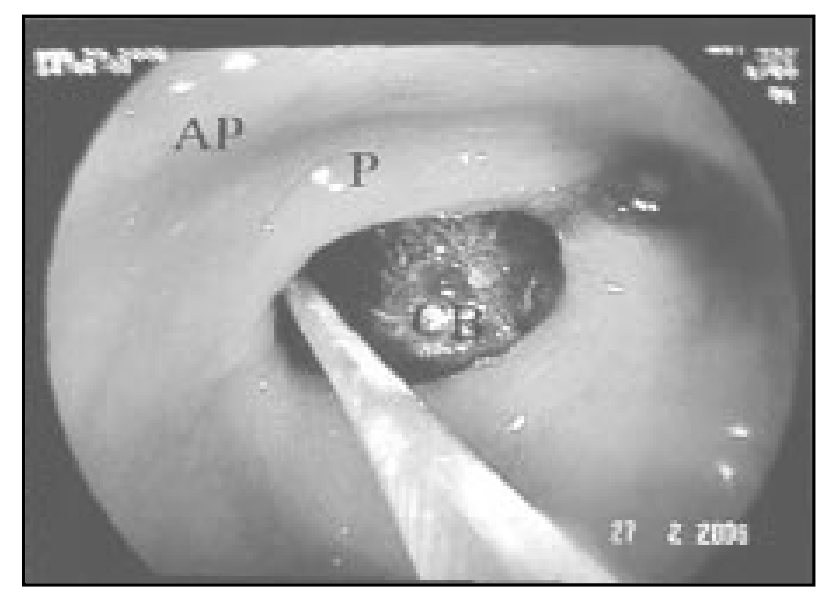

de resolución quirúrgica como en el caso de resolución endoscópica.

\section{Discusión}

La patología biliar es una enfermedad frecuente en nuestro país, es así como $20 \%$ a $30 \%$ de la población adulta presenta colelitiasis ${ }^{6,7}$. El íleo biliar es una complicación poco habitual de la patología biliar, ocurre en 0,3\%-4\% de los pacientes con colelitiasis ${ }^{8}$ y es siete veces más frecuente en mayores de 70 años ${ }^{3}$. En el íleo biliar clásico, el cálculo se impacta con más frecuencia en el íleon distal (73\%-90\%) y con menos frecuencia en el colon $(3 \%-25 \%)$ y duodeno $(3 \%-10 \%)^{1,3,4}$. El paso del cálculo ocurre a través de una fístula colecistoentérica, siendo más frecuente la colecistoduodenal (53\%-68\%) y menos frecuentemente la colecistocolónica $(5 \%)^{3,4}$.

El síndrome de Bouveret es muy poco frecuente y afecta fundamentalmente a mujeres, mayores de 50 años; cerca de $60 \%$ de los enfermos comunicados en la literatura tiene una patología asociada 3,9,10. La presentación clínica es variable e inespecífica, siendo la sepsis ${ }^{5}$ un hecho inhabitual, los síntomas se inician en promedio 5 a 7 días antes de la consulta3,4. A pesar de que $43 \%$ a $64 \%$ de los enfermos tiene antecedentes de cólicos biliares, ictericia o colecistitis aguda reciente ${ }^{3,4}$, también puede ser la primera manifestación de la colelitiasis, tal como se observó en dos enfermos de esta serie. El dolor abdominal es frecuente, generalmente se ubica en el epigastrio o en el hipocondrio derecho y su intensidad no se comelaciona con las alteraciones anatómicas subyacentes, hecho también observado en los pacientes del grupo en estudio. Menos frecuente es la hemorragia digestiva secundania a erosión duodenal, la expulsión de un cálculo biliar durante un vómito o la presencia de lesiones esofágicas asociadas a emesis intensa ${ }^{11-14}$. Dadas las caractenísticas clínicas del cuadro y debido a que la mayonía de los enfermos son mayores, es necesario descartar otras causas más frecuentes de obstrucción del vaciamiento gástrico, como el cáncer gástrico o la estenosis péptica.

El estudio de laboratorio puede mostrar leucocitosis, alteraciones hidroelectrolíticas, ácido-base y de la función renal. La magnitud de ellas dependerá de las enfermedades asociadas, el grado de respuesta inflamatoria y de los mecanismos compensatorios de cada individuo. Con menor frecuencia puede haber alteraciones de las pruebas hepáticas y elevación de la amilasa plasmática ${ }^{3,15}$, hecho que no se presentó en este grupo de pacientes.

Según lo descrito en la literatura y en este estudio, la radiografía de abdomen simple es poco útil en el diagnóstico del síndrome de Bouveret, porque la mayoría de las veces muestra signos inespecíficos y sólo en 10\% a 35\% de los enfermos se puede observar la tríada de $\operatorname{Rigler}^{4,16}$.

La ecografía también presenta limitaciones que están determinadas por las alteraciones anatómicas, la distensión intestinal y el aire o el colapso de la vesícula15-17.

La tomografía computada sugiere el diagnóstico en $75 \%$ de los casos, ya que puede visualizar mejor los elementos de la tríada de Rigler ${ }^{12}$. En esta serie, el uso de contraste oral ayudó a visualizar en forma 
directa la fístula o planteó la sospecha cuando hubo contraste en la vesícula biliar. Sin embargo, en $15 \%$ a $25 \%$ de los enfermos estudiados con tomografía computada no es posible observar el cálculo 15,16,18. En estos pacientes o en aquellos con emesis intensa y mala ingesta del medio de contraste oral, la colangiopancreatografía por resonancia magnética podńa tener un rol en el diagnóstico, porque permitináa observar con mayor precisión la anatomía, sin requenir contraste oral $^{16}$. Sin embargo hay que considerar su costo y la limitada disponibilidad a nivel nacional.

El principal objetivo del tratamiento es la extracción del cálculo para superar la obstrucción, la que se puede realizar por vía endoscópica o quirúrgica clásica ${ }^{19-21}$. La extracción endoscópica tiene un importante rol en esta patología porque es menos invasiva y se asocia a menos complicaciones, pero es difícil y requiere de endoscopistas entrenados en procedimientos terapéuticos. Podría ser más exitoso en pacientes seleccionados con cálculos de tamaño intermedio y relativamente móviles ${ }^{20,22}$. En ellos se debe evaluar después del procedimiento, el desarmollo de un íleo mecánico, como consecuencia de la migración de fragmentos hacia distal ${ }^{17}$. Los pacientes con cálculos grandes o impactados estrechamente, probablemente serán mejores candidatos a una resolución quirúrgica. En los pacientes de esta serie, la endoscopia fue una hemamienta diagnóstica y terapéutica, al diagnosticar la enfermedad en 3 enfermos y tratar en forma definitiva a uno de ellos. También se ha utilizado, pero con menos frecuencia, la litotripsia electrohidráulica endoscópica, la litotripsia endoscópica con láser y la litotripsia extracorpórea $^{23-25}$, las que necesitan equipos de alto costo y pueden requerir más de un procedimiento ${ }^{1}$.

El tratamiento clásico es la cirugía convencional. Durante la operación se debe revisar todo el intestino porque $16 \%$ de los enfermos presentan otros cálculos de ubicación variable a lo largo del intestino $^{3}$. Si es posible, es útil desplazar el cálculo al estómago, para luego realizar la extracción a través de una gastrotomía y efectuar el cierre o la

\section{REFERENCIAS}

1. Langhorst J, Schumacher B, Deselaers T, Neuhaus H. Successful endoscopic therapy of a gastric outlet obstruction due to a gallstone whit intracorporeal reparación sobre un tejido sano. En los pacientes en los que esta maniobra no es posible, se deberá realizar una duodenotomía en la cara anterior, poniendo especial énfasis en el cierre para evitar la estenosis ${ }^{3}$. A la reparación de la fístula se puede asociar la colecistectomía, sin embargo en el contexto general de un íleo biliar se ha descrito que esta podría aumentar la morbilidad y mortalidad, siendo preferible diferir estos procedimientos para un segundo tiempo quirúrgico $4,26,27$.

$\mathrm{Si}$ bien no existen evidencias con respecto al mejor tratamiento quirúrgico para los pacientes con síndrome de Bouveret, la experiencia descrita ${ }^{28} \mathrm{y}$ los hallazgos de esta serie, sugieren considerar seriamente no asociar la colecistectomía y la reparación del defecto fistuloso en un tiempo, e incluso se deberá evaluar el no realizarlas en un segundo tiempo en pacientes de edad avanzada y con múltiples patologías asociadas. Esto debe ser analizado caso a caso después de evaluar los riesgos operatorios y las expectativas de vida del enfermo.

En esta serie se realizó la colecistectomía y la reparación de la fístula en un tiempo en una mujer joven, sin enfermedades asociadas y de bajo riesgo quirúrgico.

En los casos en que se decide no realizar la colecistectomía en la primera intervención, el seguimiento endoscópico muestra que puede existir una evolución variable. En algunos casos se observó el cierre espontáneo de la fístula entre los 30 a 60 días ${ }^{29}$ y en otros, ésta permaneció activa a los 90 días de seguimiento ${ }^{1}$. Por esta razón, algunos autores aconsejan una conducta quinúrgica más agresiva en un segundo tiempo, si se considera el hecho de que existe patología biliar recurrente en 5\%-17\% de ellos 3,4,17,27, caracterizados por nuevos episodios de íleo biliar, colecistitis aguda, colangitis y el desamollo de tumores en la vesícula. Es importante considerar que en nuestro medio, $5 \%$ de los pacientes con colelitiasis desarrollan un cáncer vesicular, y que el tiempo estimado para su aparición desde el desamollo de displasia es de aproximadamente 15 años ${ }^{30,31}$.

laser lithotripsy: a case of Bouveret's syndrome. Gastrointest Endosc 2000; 51: 209-13.

2. Bouveret L. Stènose du pylore adhèrent á la vesicule. Rev Med (Paris) 1896; 16: 1-16.

3. Ciavien P, Richon J, Burgan S. Gallstone ileus. Br J Surg 1990; 77: 737-42. 
4. Kasahara $\mathrm{Y}$, Umemura $\mathrm{H}$, Shiraha S, Kuyama $\mathrm{T}$, SaкATA K, KuвотA H. Gallstone ileus: Review of 112 patients in the Japanese literature. Am J Surg 1980; 140: 437-40.

5. Bone R, Balk R, CerRa F. American College of Chest Physicians/Society of Critical Care Medicine Consensus Conference: Definitions for sepsis and organ failure and guidelines for the use of innovative therapies in sepsis. Crit Care Med 1992; 20: 864-74.

6. Roa I, Araya J, Wistuba I, Viшjaseca M, De AretxabaLA X. Litiasis de la vesícula biliar en la IX región. Estudio de autopsias en una zona con alta proporción de población mapuche. Rev Méd Chile 1991; 119: 1367-71.

7. Medina E, Pascual J, Medina R. Frecuencia de la litiasis biliar en Chile. Rev Méd Chile 1983; 111: 668-75.

8. HeinRich D, Meier J, WeHrL H, BüHLer H. Upper gastrointestinal haemorrhage preceding development of Bouveret's syndrome. Am J Gastroenterol 1993; 5: 777-80.

9. Frattaroli F, Reggio D, Guadalaxara A, Ilomei G, Lomato D, Pappalardo G. Bouveret's syndrome: case report and review of the literature. Hepatogastroenterology 1997; 44: 1019-22.

10. Rojas J, Cabané P, Hernández J, Díaz C, Vidal A. Síndrome de Bouveret. Caso clínico y revisión de la literatura. Rev Chil Cir 2006; 67: 508-10.

11. Kurtz R, Heimann T, Beck A, Kurtz A. Patterns of treatments of gallstone ileus over a 45-year period. Am J Gastroenterol 1985; 80: 95-8.

12. ShaLowitz J. Gallstone emesis. Am J Gastroenterol 1989; 84: 334-6.

13. McMahon A, McIntyre R, Rogers P, Tankel $H$. Duodenal obstruction (Bouveret's syndrome) and jaundice due to a single large gallstone. Br J Clin Pract 1990; 44: 638-9.

14. Modi B, Owens C, Ashiey S, Colson Y. Bouveret meets Boerhaave. Ann Torac Surg 2006; 81: 1493-5.

15. Brennan G, Rosenberg R, Arora S. Bouveret Syndrome. Radiographics 2004; 24: 1171-5.

16. Pickhardt P, Friediand J, Hruza D, Fisher A. CT, MR cholangiopancreatography, and endoscopy findings in Bouveret's syndrome. AJR 2003; 180: 1033-5.

17. Ariche A, Czeiger D, Gortzak Y, Shaked G, Sheigf I, LEVY I. Gastric outlet obstruction by gallstone: Bouveret Syndrome. Scand J Gastroenterol 2000; 35; 781-3.

18. Pickhardt P, Bhalla S, Balfe D. Acquired gastrointestinal fistulas: classification, etiologies, and imaging evaluation. Radiology 2002; 224: 1123-7.

19. GROve O. Acute pyloric obstruction by gallstone: report of a case diagnosed by gastroscopy. Gastrointest Endosc 1976; 22: 212-3.

20. Bedogni G, Contin S, Meinero M, Pedrazzol C, Piccinin G. Pyloroduodenal obstruction due to a biliary stone (Bouveret's syndrome) managed by endoscopic extraction. Gastrointest Endosc 1985; 31: 36-8.

21. De la Fuente H, Guzmán S, Luanos O, Ross M. Obstrucción duodenal por cálculo biliar (síndrome de Bouveret). Caso Clínico. Rev Méd Chile 1989; 117: 785-8.

22. Schneider M, Matek W, Bauer R, Domshke W. Mechanical lithotripsy of bile duct stones in 209 patients: effect of technical advances. Endoscopy 1988; 20: 248-53.

23. Moriai T, Hasegawa T, Fuzita M, Kimura A, Tani $T$, MAKINo I. Successful removal of massive intragastric gallstones by endoscopic electrohydraulic lithotripsy and mechanical lithotripsy. Am J Gastroenterol 1991; 86: 627-9.

24. Dumonceau J, Delhaye M, Devierè J, Baize M, Cremer M. Endoscopic treatment of gastric outlet obstruction caused by gallstone (Bouveret's syndrome) after extracorporeal shock wave lithotripsy. Endoscopy 1997; 29: 319-21.

25. Holl J, Sackmann M, HofFmann R, SchüsSier $P$, SAUERBRUCH T, JÜNGST D ET AL. Shock-wave therapy of gastric outlet syndrome caused by a gallstone. Gastroenterology 1989; 97: 472-4.

26. Rodríguez-San Juan J, Casado F, Fernández $M$, Morales J, Naranjo A. Cholecystectomy and fistula closure versus enterolithotomy alone in gallstone ileus. Br J Surg 1997; 84: 634-7.

27. ReISNer R, COHEN J. Gallstone ileus: a review of 1001 reported cases. Am Surg 1994; 60: 441-6.

28. Liew V, Layani L, Speakman D. Bouveret's Syndrome in Melbourne. ANZ J Surg 2002; 72: 161-3.

29. Carlson E, Gates CY, Novacovic G. Spontaneous fistulas between the gallbladder and gastrointestinal tract. Surg Gynecol Obstet 1955; 101: 321-30.

30. De Aretxabala X, Riedeman P, Burgos L, Roa I, Araya J, Echeverría X et al. Cáncer de la vesícula biliar. Estudio de casos y controles. Rev Méd Chile 1995; 123: 581-6.

31. De AretXabala X, Roa I, Burgos L. Cáncer de la vesícula biliar. Algunas consideraciones. Rev Méd Chile 1996; 124: 732-9. 